and Clyde differ by socioeconomic status and by calendar time. The children were inspected in 2006/7, 2007/8, 2008/9 and in 2009/10. A mean $d_{3} m f t$ score was created for the four posterior teeth in the upper arch and also for the two central incisors in the upper arch. The difference between the anterior and posterior scores was calculated. Additionally, an endpoint of the occurrence of obvious caries experience in both the anterior and posterior teeth was created for the upper arch. The analyses were repeated within the fifths of the Scottish Index of Multiple Deprivation. We sampled a total of 10022 children. The anterior-posterior difference was statistically greater for the most deprived children vs the most affluent children; adjusted mean difference of differences $=0.03(0.02,0.05), p<0.001$. These differences have reduced with calendar time, and by $2009 / 10$ there was no anterior-posterior difference (mean $=0.00$ for both the most deprived and the most affluent children). Over the four year period, there was more caries in both the anterior/posterior teeth for deprived children (6\%) than in affluent children (1\%); $\mathrm{OR}=5.76$. This effect was also reduced by $2009 / 10$. There is evidence that the pattern of decay in the upper arch is different in more deprived communities, with more caries in the anterior teeth and more decay in both the posterior/anterior teeth simultaneously. These effects were reduced by calendar time as population prevalence reduced.

\section{P2-184 MEASURES OF HEALTH INEQUALITY APPLIED TO DENTAL INSPECTION DATA}

doi:10.1136/jech.2011.142976j.19

${ }^{1} \mathrm{~A} D$ McMahon, ${ }^{*}{ }^{2} Y$ Blair, ${ }^{2} \mathrm{D}$ R McCall, ${ }^{1} \mathrm{~L}$ M D Macpherson. ${ }^{1}$ Glasgow University Dental School, Glasgow, Scotland, UK; ${ }^{2}$ NHS Greater Glasgow and Clyde, Glasgow, Scotland, UK

The aim of this study was to apply tests of inequality to dental inspection data from 3-year-old children in Greater Glasgow and Clyde and to compare and contrast their usefulness. Dental inspections were repeated from $2006 / 7$ to 2009/10. The measures of caries experience used were $\mathrm{d}_{3} \mathrm{mft}>0$ and mean $\mathrm{dmft}$, while the measure of socioeconomic status was "quintiles" of the Scottish Index of Multiple Deprivation. Analyses of each year's data included: raw summaries by SIMD score; logistic regression (LR) models with ROC plots and cindex; Significant Caries Index (SIC); Population Attributable Risk (PAR); Concentration Index; Gini Coefficient and Lorenz Curves; Slope Index of Inequality (SII) and Relative Index of Inequality (RII). We inspected 10022 three year old children (19\% of the population). The prevalence of obvious decay was $26 \%, 25 \%, 18 \%$ and $17 \%$, in the 4 years. Raw analyses revealed a drop in absolute inequality, but little change in relative inequality. The SII values over the 4-year period were $-1.61,-1.38,-1.16$ and -0.54 , respectively. ORs from the LR models provide relative estimates (backed up by the ROC plots), the SIC and the PAR are good at picking up the absolute reduction, the Concentration Index does not add much information, the GINI coefficient does pick up inequality but is counter-intuitive in this context, and the SII and RII are useful. We found that standard analyses such as LR and ROC are good for estimating dental inequalities, but other methods are needed to more graphically demonstrate reductions in absolute inequality.

\section{P2-185 IS THE RISE IN OBESITY PREVALENCE IN ENGLAND AND WALES FLATTENING?}

doi:10.1136/jech.2011.142976j.20

\footnotetext{
${ }^{1,2} \mathrm{~K}$ McPherson, ${ }^{*} \mathrm{~T}$ Marsh, ${ }^{2} \mathrm{M}$ Brown. ${ }^{1}$ University of Oxford, Oxford, UK; ${ }^{2}$ National Heart Forum, London, UK
}

Introduction Estimates suggest that by 2020 around 40\% of adults will be obese, this is likely to cause a serious rise in related morbidity and mortality and health service cost. This work examines the Health Survey for England from 1993 to 2008, to test for a recent change in the apparently inexorable rise in the prevalence of obesity.

Methods We compared these projections using methods developed for the Foresight Tackling Obesities programme for 2020 using eight years of survey data from 1993 to 2000 inclusive and compared them with projections using data from 2001 to 2008 We tested for a difference between the two projections and for a difference from the recent projections to the observed prevalence in 1993.

Results All eight groups have a lower projected rate of obesity in 2020 from the recent data than from the previous 8 years. All of these projections are well above the rate observed in 1993. Most notably children are recently becoming more obese at a slower rate while males aged 21-40 seem to have uniquely overcome their rising obesity in recent years.

Conclusion There is evidence for a flattening of obesity growth, at this stage only among the young. These data are cross sectional and inferring future cohort effects is less reliable, but obviously if the trends currently observable among the young represent lasting effects among cohort then the projections in the very long term made by Foresight may have been too pessimistic.

\section{P2-186 MATERNAL USE OF VITAMINS DURING PREGNANCY AND THE RISK OF TYPE 1 DIABETES IN OFFSPRING}

doi:10.1136/jech.2011.142976j.21

${ }^{1} \mathrm{~A}$ Memon, ${ }^{*} \mathrm{~S}$ Polack, ${ }^{3} \mathrm{M}$ Al-Khawari, ${ }^{3} \mathrm{M}$ Oabazard, ${ }^{4} \mathrm{~A}$ Suresh. ${ }^{1}$ Brighton and Sussex Medical School, Brighton, UK; ${ }^{2}$ London School of Hygiene and Tropical Medicine, London, UK; ${ }^{3}$ Ministry of Health, Kuwait, Kuwait; ${ }^{4}$ Faculty of Medicine, Kuwait University, Kuwait, Kuwait

Introduction It is estimated that incidence of type 1 diabetes (T1D) will double between 2005 and 2020 in most countries. In recent decades, Kuwait has experienced substantial increase in the incidence of T1D (from 4.0/100000 in 1980-1981 to 21/100000 in1992-1997). We conducted a population-based case-control interview study in Kuwait to examine antenatal/perinatal factors that may be associated with the risk of T1D in the offspring.

Methods 376 case-control pairs (aged 0-19years) individually matched on age, gender, nationality, and district of residence.

Results Univariate conditional logistic regression analysis showed that maternal age $\geq 25$ years $(\mathrm{OR}=1.8,95 \%$ CI 1.3 to 2.6), paternal age $\geq 30$ years $(2.1,1.5$ to 2.9$)$, gestational diabetes (1.8, 1.1 to 3.2$)$, birth order $\geq 3$ (2.3, 1.4 to 3.7$)$, having $>5$ siblings (1.9, 1.1 to 2.1$)$, diabetes in sibling(s) (8.3, 2.9 to 23.2), autoimmune disease in mother $(1.7,1.1$ to 2.5$)$ and congenital malformation in the family (3.9, 2.1 to 7.1$)$ were associated with a significantly increased risk of T1D; whereas maternal use of coffee, analgesics and vitamins were significantly protective. There was no association with parental consanguinity, parity, gestational age, caesarean delivery, or birth weight. In the multivariate analysis, birth order $\geq 4$ (4.5, 1.6 to 12.3 ) and maternal use of vitamins during the pregnancy $(0.2,0.1$ to 0.3$)$ remained independently associated with T1D.

Conclusion A host of factors in the antenatal/perinatal period influence the risk of T1D in the offspring. Overall, the study presents a profile of increased risk with increasing age of parents, birth order, and number of siblings. The novel finding for a protective effect of maternal use of vitamins during pregnancy warrant further investigation. 